\title{
Aula expositiva dialogada: um relato sobre essa abordagem em uma campanha do outubro rosa
}

\author{
Dialogue exhibition lesson: a report about this approach in a pink october campaign \\ Lección de exposición de diálogo: um informe sobre este enfoque em uma campaña de \\ octubre rosa
}

Catarina Maria Nogueira de Oliveira Sediyama ${ }^{1 *}$, Mariana de Moura e Dias ${ }^{1}$, Érica Toledo de Mendonça ${ }^{1}$, Pedro Alexandre Henrique Pedretti ${ }^{1}$, Diego Henrique Silveira Ramos ${ }^{1}$, Emanoela Guimarães de Castro'.

\section{RESUMO}

Objetivo: Avaliar o potencial de contribuição da aula expositiva dialogada na consolidação de conhecimentos sobre o câncer de mama entre profissionais que atuam em áreas de saúde da rede hospitalar. Relato de experiencia: Realizou-se uma palestra, e uma atividade prática com profissionais de saúde havendo aplicação de um questionário com perguntas especificas sobre o câncer de mama antes e a após o evento. Em ambas as atividades se utilizou a metodologia de aula expositiva dialogada, de forma a ouvir os participantes, estimulando-os a construir conhecimento com base em suas vivências previas. Observou-se que o conhecimento prévio dos profissionais envolve principalmente as temáticas de fatores de proteção e de diagnóstico precoce. Após a intervenção houve construção de conhecimento por parte dos alunos, haja vista que houve um maior acerto das questões, destacando-se o aumento significativo de acertos em relação a conhecimentos sobre os fatores de risco do CM. Considerações finais: A metodologia utilizada foi efetiva para a construção de conhecimento dos profissionais de saúde, o que é fundamental para formação de profissionais mais humanos e mais atentos ao diagnóstico precoce do câncer de mama.

Palavras-chave: Outubro rosa, Câncer de mama, Ensino, Metodologia ativa.

\begin{abstract}
Objective: To evaluate the contribution potential of the expository class discussed in the consolidation of knowledge about breast cancer among professionals who work in health areas of the hospital network. Experience report: There was a lecture and a practical activity with health professionals, with the application of a questionnaire with specific questions about breast cancer before and after the event. In both activities, the dialogue lecture methodology was used, in order to listen to the participants, encouraging them to build knowledge based on their previous experiences. It was observed that the professionals' prior knowledge mainly involves the themes of protective factors and early diagnosis. After the intervention, there was knowledge construction on the part of the students, given that there was a greater accuracy of the questions, highlighting the significant increase in accuracy in relation to knowledge about the risk factors of CM. Final considerations: The methodology used was effective for the construction of knowledge of health professionals, which is fundamental for the formation of more humane professionals, more attentive to the early diagnosis of breast cancer.
\end{abstract}

Key words: Pink october, Breast cancer, Teaching, Active methodology.

\section{RESUMEN}

Objetivo: Evaluar el potencial de contribución de la clase expositiva discutida en la consolidación del conocimiento sobre el cáncer de mama entre los profesionales que laboran en las áreas de salud de la red hospitalaria. Informe de experiencia: Se realizó una charla y una actividad práctica con profesionales de la salud, con la aplicación de un cuestionario con preguntas específicas sobre el cáncer de mama antes y

1 Universidade Federal de Viçosa, Viçosa - MG.

*E-mail: catynogueira@yahoo.com.br 
después del evento. En ambas actividades se utilizó la metodología de charla de diálogo, con el fin de escuchar a los participantes, animándolos a construir conocimientos a partir de sus experiencias previas. Se observó que el conocimiento previo de los profesionales involucra principalmente los temas de factores protectores y diagnóstico precoz. Tras la intervención, se construyó conocimiento por parte de los estudiantes, dado que hubo un mayor acierto en las preguntas, destacando el aumento significativo de aciertos en relación al conocimiento sobre los factores de riesgo de MC. Consideraciones finales: La metodología utilizada resultó efectiva para la construcción de conocimientos de los profesionales de la salud, lo cual es fundamental para la formación de profesionales más humanos, más atentos al diagnóstico precoz del cáncer de mama.

Palabra clave: Octubre rosa, Cáncer de mama, Docencia, Metodología activa.

\section{INTRODUÇÃO}

Para o ano de 2020, o Instituto Nacional do Câncer (INCA) (2019) estima aproximadamente 70.000 novos casos de Câncer de Mama (CM) no Brasil, representando quase $30 \%$ de todas as neoplasias que acometem o sexo feminino. Além disso, em 2018, o Brasil assumiu o segundo lugar entre os países com a maior incidência de CM, com 62,9 casos por 100 mil mulheres (INCA 2019).

Dentre os fatores de risco do $\mathrm{CM}$, destacam-se a idade avançada e características relacionadas a vida reprodutiva da mulher como menarca precoce, menopausa tardia, reposição hormonal pós-menopausa, paridade e aleitamento materno (BRASIL 2013). Contudo, fatores ambientais e de estilo de vida, como sedentarismo, obesidade, ingestão de bebida alcóolica e exposição à radiação ionizante também influenciam o desenvolvimento dessa neoplasia (OHL ICB, et al., 2016).

A atuação dos profissionais de saúde, através de atividades de orientação e educação sobre saúde da mulher, é fundamental para a prevenção primária do câncer de mama haja vista que a promoção de informação pode reduzir a exposição a fatores risco ambientais (FALKENBERG MB, et al., 2014; OHL ICB, et al., 2016).

Além disso, a prevenção secundária pode ser realizada através do exame clínico das mamas e da mamografia, sendo essa temática amplamente estimulada nas campanhas Outubro Rosa, realizadas mundialmente todos os anos (OHL ICB, et al., 2016). A importância dessas medidas inclusive, é normatizada pelo Ministério da Saúde e deve ser buscada em todos os níveis de atenção, visando oferecer à mulher a oportunidade do diagnóstico precoce, o que tanto melhora a chance de sucesso do tratamento quanto 0 prognóstico (GUTIÉRREZ MGV e ALMEIDA AM, 2017).

A capacitação de profissionais de saúde para o diagnóstico precoce do CM e para efetiva execução de programas de rastreamento populacional é necessário e importante, destacando-se o uso de aula expositiva dialogada como metodologia de ensino. Ao contrário da aula expositiva tradicional, onde o aluno apenas escuta o conteúdo, na aula expositiva dialogada, o aluno é estimulado a participar das discussões, de forma a utilizar conhecimentos prévios para construção dos novos conceitos abordados em sala de aula (LOPES TO, 2012).

Diante do exposto, o presente trabalho visou avaliar o potencial de contribuição da aula expositiva dialogada na consolidação de conhecimentos sobre o CM entre profissionais que atuam em áreas de saúde da rede hospitalar de uma cidade no interior de Minas Gerais.

\section{RELATO DE EXPERIÊNCIA}

Durante uma campanha do Outubro Rosa, professores e estudantes dos cursos de Enfermagem e Medicina, de uma Universidade Pública Federal, realizaram uma intervenção educativa com profissionais de duas instituições de saúde do município de Viçosa-MG.

As atividades foram realizadas nas próprias instituições de saúde, de modo a estimular a participação do maior número possível de funcionários. Além disso, todos os profissionais de saúde, presentes no dia da intervenção, foram convidados a participar das atividades, não havendo critérios de exclusão para a participação. 
Destaca-se que antes de iniciar a atividade, esclareceu-se para os profissionais que aquela atividade fazia parte de uma campanha educativa sobre o câncer de mama e que haveria aplicação de um questionário em 2 momentos. Logo, todos os participantes foram convidados a participar das atividades. Os funcionários que não concordaram em responder ao questionário puderam assistir à palestra e também participaram das discussões e da atividade prática. Contudo, para os que concordaram em participar houve aplicação dos questionários em 2 momentos: antes das discussões e após ela. Os funcionários que possuíam dificuldades com relação à escrita e/ou leitura tiveram ajuda de voluntários para responder ao questionário.

As atividades realizadas incluíram a apresentação de uma palestra educativa, uma atividade prática e a aplicação de um questionário com apenas 5 perguntas, referentes as temáticas abordadas na palestra e na atividade prática.

A palestra foi sobre aspectos relacionados ao câncer de mama, abordando os principais sinais e sintomas da doença, seus métodos de rastreamento populacional, diagnóstico e tratamento. Durante essa explanação, os participantes foram estimulados a interromper os participantes, relatando suas experiências e angústias em relação as temáticas abordadas, havendo uma rica discussão tanto entre participantes e palestrantes, como também entre os próprios participantes. Além disso, em caso de dúvidas, imediatamente houve a resposta dos questionamentos realizadas, observando-se que essa abertura para "interromper" a explanação foi fundamental para que os profissionais de saúde se sentissem confortáveis para discutir os temas abordados, gerando um amplo debate, com construção de conhecimentos.

Em seguida, durante a atividade prática, foram apresentados aos participantes, modelos de mamas com e sem alterações relacionadas ao câncer. Todos os modelos foram previamente construídos pelos estudantes de medicina e enfermagem, que agora estavam discutindo as possíveis alterações fenotípicas com os profissionais de saúde.

O objetivo foi que, durante a observação e manipulação dos modelos, houvesse uma abordagem dialógica com aspectos chave sobre o tema proposto, como fatores de risco e de prevenção ao $\mathrm{CM}$, bem como se capacitasse os profissionais para identificação de alterações nas mamas. Visto que novamente houve amplo espaço para participação dos profissionais de saúde, acredita-se que esse objetivo tenha sido alcançado.

Para avaliar os conhecimentos prévios sobre o tema e o aproveitamento da atividade educativa, foi aplicado um questionário com 5 questões sobre o $\mathrm{CM}$ antes e após a conclusão das demais atividades (palestra, atividade prática e discussão). Questionou-se os participantes sobre os fatores de risco do CM, atividades de prevenção ao $\mathrm{CM}$, diagnóstico precoce, realização de mamografia e alterações nas mamas.

Antes da intervenção, a questão relacionada a fatores protetores do $\mathrm{CM}$ foi a que apresentou maior número de acertos pelos participantes e a questão que abordava a importância do diagnóstico precoce foi a que apresentou maior desconhecimento por parte dos participantes. Além disso, os temas de maior conhecimento pré-teste foram os relacionados aos fatores de proteção e aos métodos de rastreamento da doença. A aplicação desse questionário foi fundamental para identificarmos quais eram os pontos críticos de diálogo que deveriam ser abordados na atividade, sendo esse direcionamento fundamental para o desenvolvimento da atividade educativa.

Por fim, após a intervenção, observamos que, em todas as questões, houve aumento no número de acerto, - que nos permitiu perceber a efetividade tanto das atividades desenvolvidas quanto do processo metodológico escolhido, que priorizou a construção de conhecimentos a partir do diálogo participativo.

\section{DISCUSSÃO}

O Outubro Rosa é um movimento de importante disseminação de conhecimento sobre o CM, sendo uma das campanhas de saúde com maior adesão pela sociedade civil (AGOSTINHO JC, et al., 2019; ASSIS M, et al., 2020). Esse movimento possibilita que as mulheres compreendam o processo de adoecimento e 0 rastreamento da doença, o que estimula a adesão aos programas de diagnóstico e prevenção do $\mathrm{CM}$, levando a impactos positivos em sua saúde (ASSIS M, et al., 2020; GUTIÉRREZ MGV e ALMEIDA AM, 2017). 
Dados mostram que as mulheres não têm conhecimento adequado sobre o $\mathrm{CM}$ e seus fatores de risco, bem como não participam de campanhas de prevenção (RODRIGUES ARS, et al., 2018). Infelizmente, também foram detectados déficits na formação de profissionais de saúde o que reforça a necessidade de melhor capacitação desses profissionais, de modo que eles possam disseminar informações corretas e assertivas dobre o câncer de mama, gerando impacto direto na vida das mulheres acometidas pelo CM, visto que quanto mais precoce o diagnóstico, maior a chance de sobrevida dessas mulheres (MORAES DC, et al., 2016; NUNES VLS, et al., 2020; OHL ICB, et al., 2016).

Segundo Nez e Santos (2017), aulas expositivas são muito utilizadas com o objetivo de transmitir um conhecimento em um curto período, e quando preparada de maneira adequada, atinge-se o objetivo de transmissão de conhecimento. Contudo, o uso dessa metodologia tradicional pode não produzir um aprendizado significativo, devendo o profissional de ensino fazer uma reflexão sobre o modelo de aprendizagem que objetiva construir (SILVA MJ, 2017).

De fato, nas aulas expositivas, o estudante não é estimulado a desenvolver um pensamento crítico, não havendo, portanto, construção de conhecimento. É um modelo antigo onde o professor é o detentor do conhecimento e o estudante é um recebedor passivo de informações (FIGUEIREDO MFS, et al., 2010).

Já no ensino ativa há um melhor aprendizado, visto que o estudante é desafiado a pensar e tem autonomia, o que faz com que o processo de ensino-aprendizagem seja mais enriquecedor (FIGUEIREDO MFS, et al., 2010; MITRE SM, et al., 2008; SMOLKA MLRM, et al., 2020). Nesse caso, o aprendizado nem sempre é percebido claramente pelos estudantes (DESLAURIERS L, et al., 2019). Contudo, esse é um modelo dialógico construtivo, onde o conhecimento prévio dos estudantes tem importância, o que tanto contribui para a participação de todos no processo, como também fortalece o conhecimento construído (FIGUEIREDO MFS, et al., 2010; MITRE SM, et al., 2008).

$\mathrm{Na}$ metodologia adotada, o método expositivo dialogado, o aluno tem um papel ativo no processo de aquisição de conhecimento, atuando como agentes de seu processo de ensino-aprendizagem (FIGUEIREDO MFS, et al., 2010; MITRE SM, et al., 2008; PAIM AS, et al., 2015).Nesse contexto, durante a apresentação da aula, buscou-se utilizar uma linguagem acessível e uma abordagem sem enfatizar a memorização do tema, ou seja, objetivou-se a construção de uma maior compreensão da doença e suas implicações na vida das pacientes (INCA 2016). Também se discutiu aspectos relacionados aos programas de rastreamento e a importância de adesão a esses programas disponíveis de maneira gratuita pela rede pública de saúde, o Sistema Único de Saúde (SUS). Além disso, a todo momento os participantes tinham a oportunidade de tirar dúvidas e questionar sobre o tema, e ao final, uma ampla discussão foi realizada, respeitando aspectos individuais e culturais dos participantes.

Após a explicação teórica do tema, utilizando estratégias da discussão dialogada, foi realizada uma oficina com os participantes, com a apresentação dos modelos de mama com e sem alterações causadas pelo CM. Esses modelos permitem que o profissional de saúde se capacite para a identificação de indícios visuais de anormalidade na mama, bem como para a identificação de alterações físicas mediante o toque nas mamas, sendo essa prática importante na detecção precoce do CM (ALVES GKO, et al., 2020; NUNES VLS, et al., 2010).

A condução da atividade teórico-prática foi realizada de maneira a levar a construção do conhecimento pelos participantes, através da troca de saberes de conhecimentos ativos e dinâmicos. Durante a realização dessa atividade, tivemos a oportunidade de realizar uma maior interação com os participantes, esclarecendo as dúvidas, e ouvindo as demandas. Os participantes também tiveram a oportunidade de expor ideias e conhecimentos preexistente sobre o CM e compartilhar experiências pessoais, contribuindo para sedimentar as informações recebidas. Com isso, buscamos construir conhecimento e informação da forma mais clara possível, acreditando que esse conhecimento construído será fundamental para que os profissionais desempenhem atividades de prevenção ao CM (INCA 2016).

O ganho de conhecimento com a Campanha do Outubro Rosa foi visualizado via o maior percentual de acerto em relação as questões realizadas antes da intervenção, destacando-se o ganho de conhecimento em 
relação aos fatores de risco para o desenvolvimento do câncer de mama ( $Q$ 01). A disseminação de conhecimento sobre os fatores de risco é uma temática chave para a prevenção do $\mathrm{CM}$, sendo um dos temas mais abordados em campanhas divulgadas na mídia brasileira (ASSIS M, et al., 2020). Além disso, o ganho de conhecimento sobre os fatores de risco torna os profissionais de saúde mais capacitados para abordar essa temática com as mulheres, o que auxilia diretamente na prevenção do CM (ALVES GKO, et al., 2020).

Apesar de abordado nas atividades e discussões, a importância do diagnóstico precoce para as pacientes foi a temática que menos demonstrou aprimoramento após a intervenção, devendo ser desenvolvidas estratégia mais específica para a abordagem desse tema. Essa é a segunda temática mais abordada em questões publicitárias relacionadas ao $\mathrm{CM}$, só perdendo para a divulgação de eventos solidários de apoio a pacientes com a doença (ASSIS M, et al., 2020), o que reforça a importância da construção de conhecimento sobre o diagnóstico precoce.

Houve diferença significante $(p=0.006)$ em relação ao conhecimento dos participantes sobre o Câncer de Mama após a aula e oficina, demonstrando aquisição de conhecimento após a realização da atividade. Esse resultado é relevante e positivo já que o conhecimento gera empoderamento, o que é a base para a mudanças de hábito e a tomada de ações capazes de transformação (INCA 2016). Além disso, a disseminação de conhecimentos que atuem na prevenção do $\mathrm{CM}$ reduz os custos relacionados ao tratamento dessa doença, sendo inteligente investir na capacitação contínua de profissionais de saúde (ALVES GKO, et al., 2020; FALKENBERG MB, et al., 2014).

A estratégia de aula expositiva dialogada associada a atividade prática, utilizando modelos de mama com ou sem lesão por $\mathrm{CM}$, contribuiu para a aquisição e construção de conhecimento em relação à aspectos relacionados ao $\mathrm{CM}$ como fatores de risco, prevenção da doença, métodos de rastreamento, importância do diagnóstico precoce e sintomas da doença. Logo, utilizar essa metodologia para a capacitação de profissionais de saúde é fundamental para efetiva transmissão de conhecimento às mulheres, de forma clara, objetiva e compreensível, o que consequentemente permite uma maior execução de ações que favoreçam um diagnóstico precoce do $\mathrm{CM}$, bem como uma maior adesão aos programas de rastreamento.

\section{REFERÊNCIAS}

1. AGOSTINHO JC, et al. Análise dos fatores de risco do Câncer de Mama e avaliação da campanha preventive "Outubro Rosa". Revista Saúde UniToledo, 2019; 3: 97-108.

2. ALVES GKO, et al. Educação em saúde e prevenção do câncer de mama no município de Itaúna, Minas Gerais. Revista Nursing, 2020; 23: 4442-4446.

3. BRASIL. Ministério da Saúde. Secretaria de Atenção à Saúde. Departamento de Atenção Básica. Controle dos cânceres do colo do útero e de mama. 2013. Disponível em: https://bvsms.saude.gov.br/bvs/publicacoes/controle_canceres_colo_utero_2013.pdf. Acessado em: 1 de maio de 2021.

4. ASSIS M, et al. Detecção precoce do câncer de mama na mídia brasileira no Outubro Rosa. Physis: Revista de Saúde Coletiva, 2020; 30: e300119.

5. DESLAURIERS L, et al. Measuring actual learning versus feeling of learning in response to being actively engaged in the classroom. Proceedings of the National Academy of Sciences of the United States of America, 2019; 116: 1925119257

6. NEZ E, SANTOS CA. Reflexões sobre a metodologia das aulas expositivas na educação básica e superior. Revista de Educação do Vale do Arinos, 2017; 4: 24-36.

7. FALKENBERG MB, et al. Educação em saúde e educação na saúde: conceitos e implicações para a saúde coletiva. Ciência e Saúde Coletiva, 2014; 19: 847-852.

8. FIGUEIREDO MFS, et al. Modelos aplicados às atividades de educação em saúde. Revista Brasileira de Enfermagem 2010; 63: 117-121.

9. GUTIÉRREZ MGV, ALMEIDA AM. Outubro rosa. Acta Paulista de Enfermagem, 2017; 30: 3-5.

10. Instituto Nacional do Câncer José Alencar Gomes da Silva (INCA) 2019. Estimativa 2020: Incidência no câncer no Brasil. 2020. Disponível em: https://www.inca.gov.br/publicacoes/livros/estimativa-2020-incidencia-de-cancer-nobrasil. Acessado em: 7 de abril de 2021.

11. LOPES TO. Aula expositiva dialogada e aula simulada: comparação entre estratégias de ensino na graduação de enfermagem. Dissertação (Mestrado) - Escola de Enfermagem. Universidade de São Paulo, São Paulo, 2012; 125p.

12. MITRE SM, et al. Metodologias ativas de ensino-aprendizagem na formação professional em saúde: debates atuais. Ciência e Saúde Coletiva, 2008; 13: 2133-2144 
13. MORAES DC, et al. Rastreamento oportunístico do câncer de mama desenvolvido por enfermeiros da Atenção Primária à Saúde. Revista da Escola de Enfermagem da USP, 2016; 50: 14-21.

14. NUNES VLS, et al. A importância da educação em saúde como forma de prevenção ao câncer de mama: Um relato de experiência em uma unidade básica de saúde de Palmas/TO. Revista Extensão 2020; 4: 108-114.

15. OHL ICB, et al. Ações públicas para o controle do câncer de mama no Brasil: revisão integrativa. Revista Brasileira de Enfermagem, 2016; 69: 793-803.

16. PAIM AS, et al. Metodologias de ensino utilizadas por docentes do curso de enfermagem: enfoque na metodologia problematizadora. Enfermería Global, 2015, 37: 153-169.

17. INSTITUTO NACIONAL DO CÂNCER (INCA). Comunicação, uma estratégia contra o câncer. Rede Câncer. 2016. Disponível em: https://www.inca.gov.br/sites/ufu.sti.inca.local/files//media/document//rrc-36-gestao-comunicacaouma-estrategia-contrao-cancer.pdf. Acessado em 20 de abril de 2021.

18. RODRIGUES ARS, et al. Câncer de mama: Conhecimento de usuárias do serviço público de saúde. Temas em saúde, 2018; 18: 5-12.

19. SILVA MJ. Abordagens tradicional e ativa: Uma análise da prática a partir da vivencia no estágio supervisionado em docência. Educere, Anais XIII Congresso Nacional de Educação. Curitiba, Paraná, Brasil, 2017.

20. SMOLKA MLRM, et al. A autonomia do estudante na educação médica. Revista da JOPIC, 2020; 3: 60-67. 